\title{
Head and neck cancer triggers the internalization of TLR3 in natural killer cells
}

\author{
LEI XIE ${ }^{1}$, RALPH PRIES $^{2}$, REBECCA KESSELRING $^{2}$, SANDRA WULFF $^{2}$ and BARBARA WOLLENBERG ${ }^{2}$ \\ ${ }^{1}$ Department of Head and Neck Surgery, Sir Run Run Shaw Hospital, Zhejiang University, 310016 Hangzhou, P.R. China; \\ ${ }^{2}$ Department of Otorhinolaryngology, University of Schleswig-Holstein Campus Lübeck, 23538 Lübeck, Germany
}

Received May 4, 2007; Accepted June 18, 2007

\begin{abstract}
Natural killer (NK) cells play a dominant role in the network of innate immunity. Via Toll-like receptor 3 (TLR3), NK cells can be efficiently stimulated by doublestranded (ds)RNA. In head and neck squamous cell carcinoma (HNSCC), NK cells seem to be strongly impaired, but the true mechanisms of immune escape are not sufficiently known to date. It is obvious that the microenvironment of head and neck cancer results in strongly affected immune functions. NK cells play a major role in the local immune response of HNSCC. In this study we showed that TLR3 is predominantly expressed on the cell surface of native NK cells and becomes rapidly internalized in response to the HNSCC microenvironment. These findings represent a novel potential immune escape mechanism of head and neck cancer. The internalization of TLR3 in response to HNSCC was also observed in fibroblasts expressing heterologous TLR3 protein. Specific stimulation of NK cell TLR3 with its ligand polyinosinic-polycytidylic acid (Poly I:C) impairs the internalization of this Toll-like receptor and leads to activated NK cells within the HNSCC microenvironment.
\end{abstract}

\section{Introduction}

Head and neck squamous cell carcinoma (HNSCC) is one of the most frequent cancers in the world, and over the last 40 years the 5-year survival rate has only marginally improved. Cells of head and neck cancer are known to develop several molecular strategies to escape from efficient immune responses. It is thought that various tumor-secreted immunosuppressive mediators contribute to massively affected immune functions within the malignant transformation process (1-3).

Natural killer (NK) cells are a component of innate immunity and play an important role in anti-infection activity

Correspondence to: Dr Barbara Wollenberg, UK-SH, Klinik für HNO, Ratzeburger Allee 160, 23538 Lübeck, Germany

E-mail: barbara.wollenberg@hno.uni-luebeck.de

Key words: natural killer cells, Toll-like receptor, head and neck cancer, tumor immunology and tumor surveillance, especially in $\operatorname{HNSCC}(4,5)$. NK cells can be triggered through various receptors depending on specific ligands presented by target cells in a given encounter (6-9). However, NK cells isolated from cancer patients exhibit strongly impaired anti-tumor functions $(10,11)$.

Recently it has been shown that treatment with the synthetic double-stranded (ds)RNA polyinosinic-polycytidylic acid (Poly I:C), a mimic of a common product of viral infections, significantly up-regulates both natural and CD16-mediated cytotoxicity of highly purified human NK cells (12). Poly I:C as well triggers the novel capability of producing CXCL10 chemokine in human NK cells and synergistically enhances interferon- $\gamma(\mathrm{IFN}-\gamma)$ production induced by either adaptive or innate cytokines (12). Poly I:C is known to be recognized by Toll-like receptor 3 (TLR3) (13), one of ten different human TLRs which recognize conserved molecular patterns to trigger innate immune responses $(14,15)$.

TLR3 stimulation was shown to rapidly up-regulate NK cell in vivo functions, whereas the NK cell ability to directly respond to dsRNA is still mostly unknown (12).

In this study we investigated the regulation and subcellular distribution of TLR3 in human NK cells with respect to the influence of the HNSCC microenvironment and Poly I:C. Among human TLR proteins we found only TLR1, TLR2, TLR3, and TLR7 to be significantly expressed in native NK cells. Protein levels of these Toll-like receptors were not affected by the microenvironment of head and neck cancer.

Flow cytometric analysis of the subcellular localization of TLR3 revealed a drastic change in the intracellular and extracellular distribution of this Toll-like receptor. TLR3 is rapidly internalized in NK cells in response to the HNSCC microenvironment.

This mechanism was also observed in fibroblastic cells expressing heterologous TLR3. HNSCC-induced NK cell alteration can be counteracted by specific TLR3 activation. Poly I:C stimulation impairs the internalization of TLR3 and leads to activated NK cells in the presence of the HNSCC microenvironment.

\section{Materials and methods}

Isolation of NK cells. Human peripheral blood mononuclear cells were isolated from buffy coats provided by the blood bank of the University of Lübeck (Lübeck, Germany). Blood 


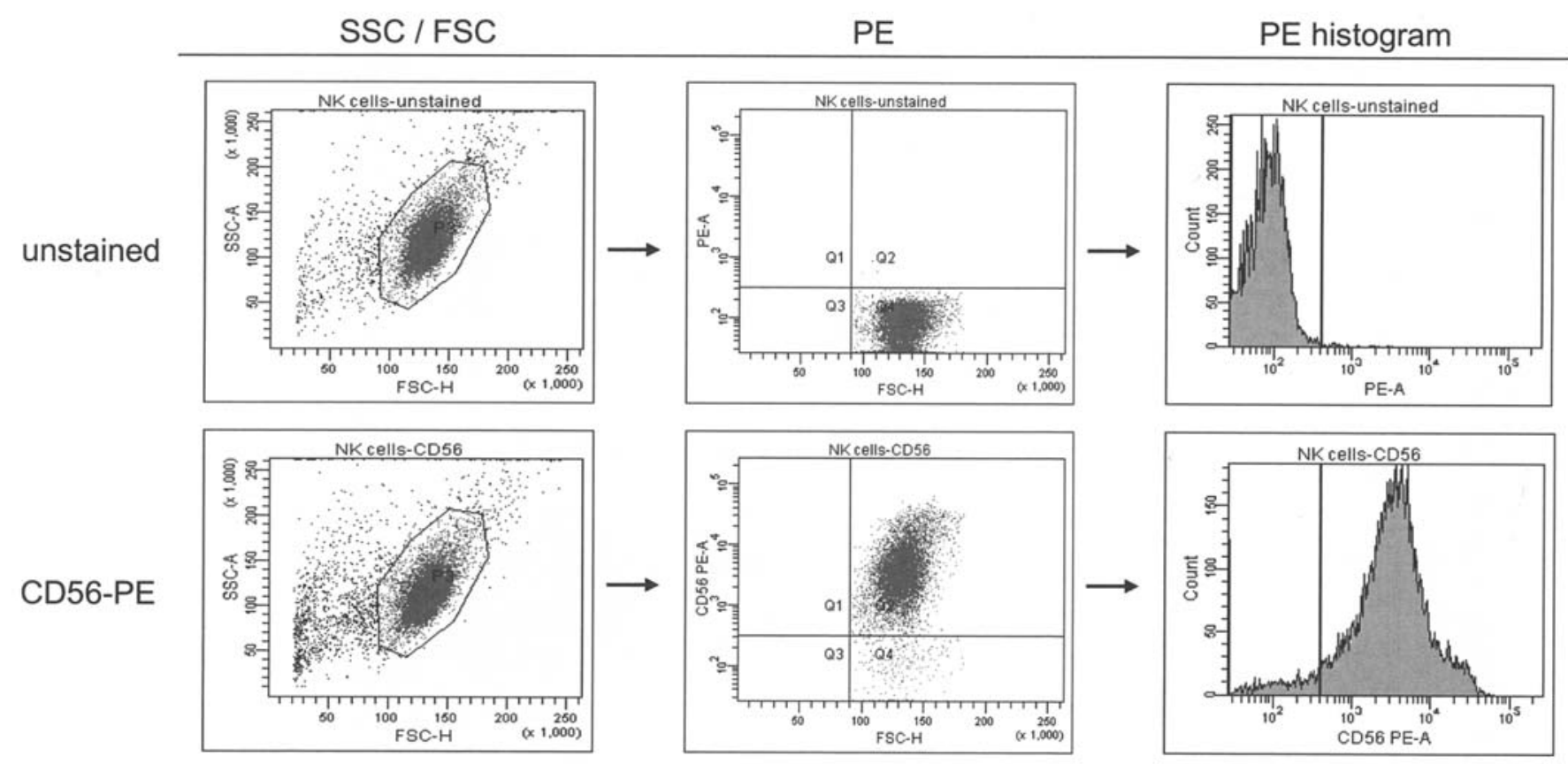

Figure 1. Flow cytometric analysis of isolated NK cells. Isolated NK cells were characterized by SSC/FSC properties and identified by the expression of the characteristic surface antigen CD56 using phycoerythrin (PE)-labeled anti-CD56 antibodies. NK cell isolation resulted in purities $>95 \%$.

donors were 18 - to 65 -year-old healthy men and women who tested negative for HIV, hepatitis B virus, and hepatitis C virus. PBMC were prepared from buffy coats by FicollHypaque density gradient centrifugation. Depletion of nonNK cells ( T cells, B cells, dentritic cells, monocytes, granulocytes and erythroid cells) was used to isolate untouched NK cells with a cocktail of biotin-conjugated antibodies against CD3, CD4, CD14, CD15, CD19, CD36, CD123, and Glycophorin A, and anti-biotin microbeads, according to the manufacturer's protocol (Miltenyi Biotec, Bergisch Gladbach, Germany).

Cell culture and preparation of supernatants. Permanent HNSCC cell lines BHY (DSMZ Germany) (16), PCI-1, and PCI-13 (hypopharyngeal cancer, Pittsburgh Cancer Institute) were used to generate HNSCC supernatants. Cells were cultured in Dulbecco's modified Eagle's medium (DMEM, Gibco) supplemented with $10 \%$ FCS, $1 \mathrm{mM}$ glutamine and $0.1 \mathrm{mM}$ sodium pyruvate. Cell-free supernatants were collected by centrifugation and filtration after $48 \mathrm{~h}$ of cell cultivation. Isolated NK cells were incubated at $37^{\circ} \mathrm{C}$ in $5 \%$ $\mathrm{CO}_{2}$ for $24 \mathrm{~h}$ in HNSCC supernatants or DMEM. For cell stimulation $50 \mu \mathrm{g} / \mathrm{ml}$ Poly I:C (Sigma) was added. Mouse fibroblasts NIH3T3 were used for transfection with plasmid pUNO-hTLR3 (Invitrogen). This plasmid encodes for human TLR3 driven from a strong hEF1-HTLV promoter. The plasmid encodes the bsr (blasticidin resistance gene) from Bacillus cereus that confers resistance to the antibiotic Blasticidin $\mathrm{S}$ and allows blasticidin selection in both mammalian cells and E. coli bacteria.

Flow cytometry. Surface antigen staining was performed as described previously (17). Cells were stained with fluorescein5-isothiocyanate (FITC)-, or phycoerythrin (PE)-conjugated antibodies (BD Biosciences). Propidium-iodide was used to identify dead cells. For intracellular staining NK cells were permeabilized with saponin buffer (PBS, 0.1\% saponin, $1 \%$ FCS, and 1 M HEPES). Samples were analyzed on a FACSCanto (BD Biosciences, Heidelberg, Germany), and data acquisition was performed using the FACS DIVA software (BD Biosciences, Heidelberg, Germany).

Protein analysis. Cell extracts were prepared and solubilized, and protein concentrations were determined using the BioRad protein assay kit (Bio-Rad $\mathrm{GmbH}$, Munich, Germany) with bovine serum albumin (BSA) as a standard. Aliquots of protein extracts $(20 \mu \mathrm{g})$ were run on $10 \%$ acrylamide SDSPAGE and were then transferred to a nitrocellulose membrane. Blots were decorated with specific antibodies (Biomol, Hamburg, Germany) as specified in the figure legends.

\section{Results}

Isolation and identification of natural killer cells. Human natural killer (NK) cells comprise about $10-20 \%$ of human peripheral blood lymphocytes. They can be identified by the expression of the characteristic surface antigen CD56, an isoform of the neural cell adhesion molecule, present on all NK cells (18). We isolated untouched NK cells from the human peripheral blood of healthy donors using magnetic bead separation, whereas a defined antibody cocktail was used for the depletion of all non-NK cells. Light microscopy was used to calculate cell numbers as well as to analyze the viability of the isolated cells by trypan-blue staining of dead cells. Isolated NK cells were characterized by flow cytometry using SSC/FSC properties and identified by the use of phycoerythrin (PE)-labeled anti-CD56 antibodies. Our data revealed purities of isolated NK cells $>95 \%$ (Fig. 1). 


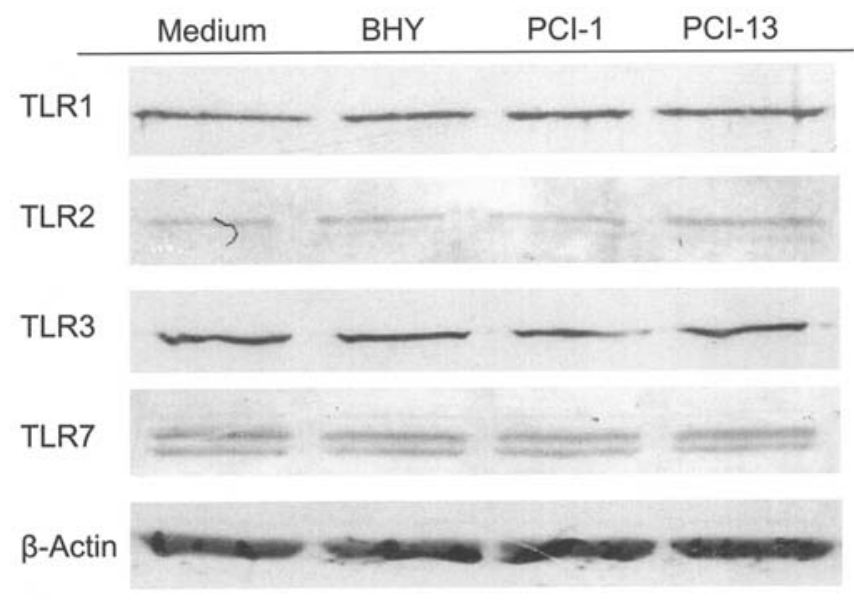

Figure 2. TLR protein expression in human NK cells. Among the analyzed TLR proteins (TLRs 1-10) only 4 TLRs were significantly expressed in native human NK cells, namely TLR1, TLR2, TLR3, and TLR7. Expression of these TLR proteins was not affected in response to $24 \mathrm{~h}$ of incubation in supernatants of three different permanent HNSCC tumor cell lines (BHY, PCI-1, and PCI-13).

TLR expression in human NK cells. Western blot experiments were carried out to examine the protein expression levels of human TLRs 1-10 with respect to the influence of the HNSCC microenvironment. Therefore, isolated NK cells were incubated in standard medium as well as in supernatants of three different permanent HNSCC tumor cell lines (BHY, PCI-1, and PCI-13) for $24 \mathrm{~h}$ and then subjected to protein analysis.

Besides TLR3, only three other TLRs were significantly expressed in native human NK cells, namely TLR1, TLR2, and TLR7 (Fig. 2). Our investigations revealed a constitutive expression of these TLR proteins in NK cells which was not affected in response to $24 \mathrm{~h}$ of incubation in different HNSCC supernatants (Fig. 2).
HNSCC removes TLR3 from the surface of $N K$ cells. To further analyze the effects of HNSCC supernatants on the regulation of TLR3 in NK cells, flow cytometry was used to distinguish between intracellular and surface expression levels of this Toll-like receptor.

Our investigations identified TLR3 as a predominantly surface-expressed receptor in native NK cells which was also found in significant levels in the cellular lumen (Fig. 3).

Surprisingly, incubation of isolated NK cells in HNSCC supernatants for $24 \mathrm{~h}$ resulted in an internalization of TLR3 (Fig. 3). TLR3 expression was rapidly down-regulated on the cell surface, and correspondingly, increased cytoplasmic protein levels were detected after incubation with HNSCC supernatants. Identical findings were achieved using supernatants of three different HNSCC cell lines (Fig. 3). The internalization of TLR3 in response to HNSCC was also observed in fibroblasts expressing heterologous TLR3 protein (Fig. 4).

Poly I:C triggers the surface expression of TLR3 in the presence of HNSCC. In order to analyze the influence of head and neck cancer on NK cell-stimulating conditions, we examined the activation of TLR3 in the presence of the immunosuppressive influence of the HNSCC microenvironment. Therefore isolated NK cells were simultaneously incubated with HNSCC supernatants and stimulated with Poly I:C.

Our data demonstrated the antagonistic effects of Poly I:C and HNSCC supernatants. Poly I:C-stimulated NK cells revealed a significantly increased surface expression of TLR3 in the presence of HNSCC (Fig. 5). Fig. 5 illustrates that Poly I:C is able to impair the HNSCC-induced internalization of TLR3.

Poly I:C activates NK cells in the presence of HNSCC. CD69 is a characteristic activation marker which is expressed on the surface of NK cells in response to various activating

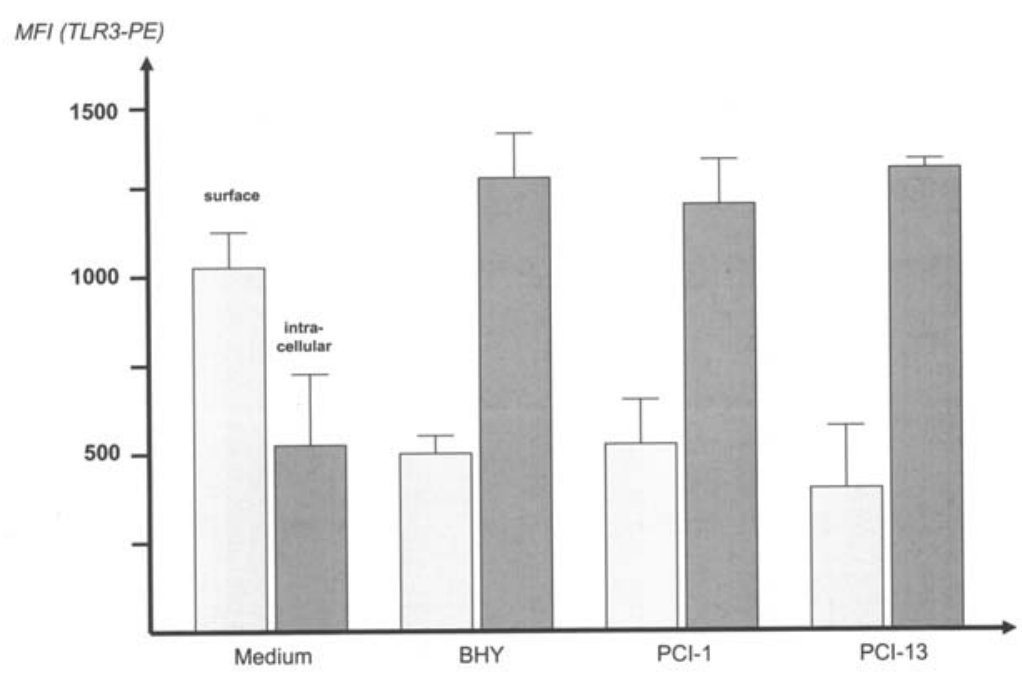

Figure 3. Subcellular distribution of TLR3 in NK cells. In native NK cells, TLR3 was predominantly expressed on the cell surface whereas a smaller fraction was found within the cellular lumen. TLR3 became rapidly internalized and removed from the cell surface in response to $24 \mathrm{~h}$ of incubation in HNSCC supernatants of three different HNSCC cell lines (BHY, PCI-1, and PCI-13). Solid bars illustrate the measured MFI (mean fluorescence intensity). 


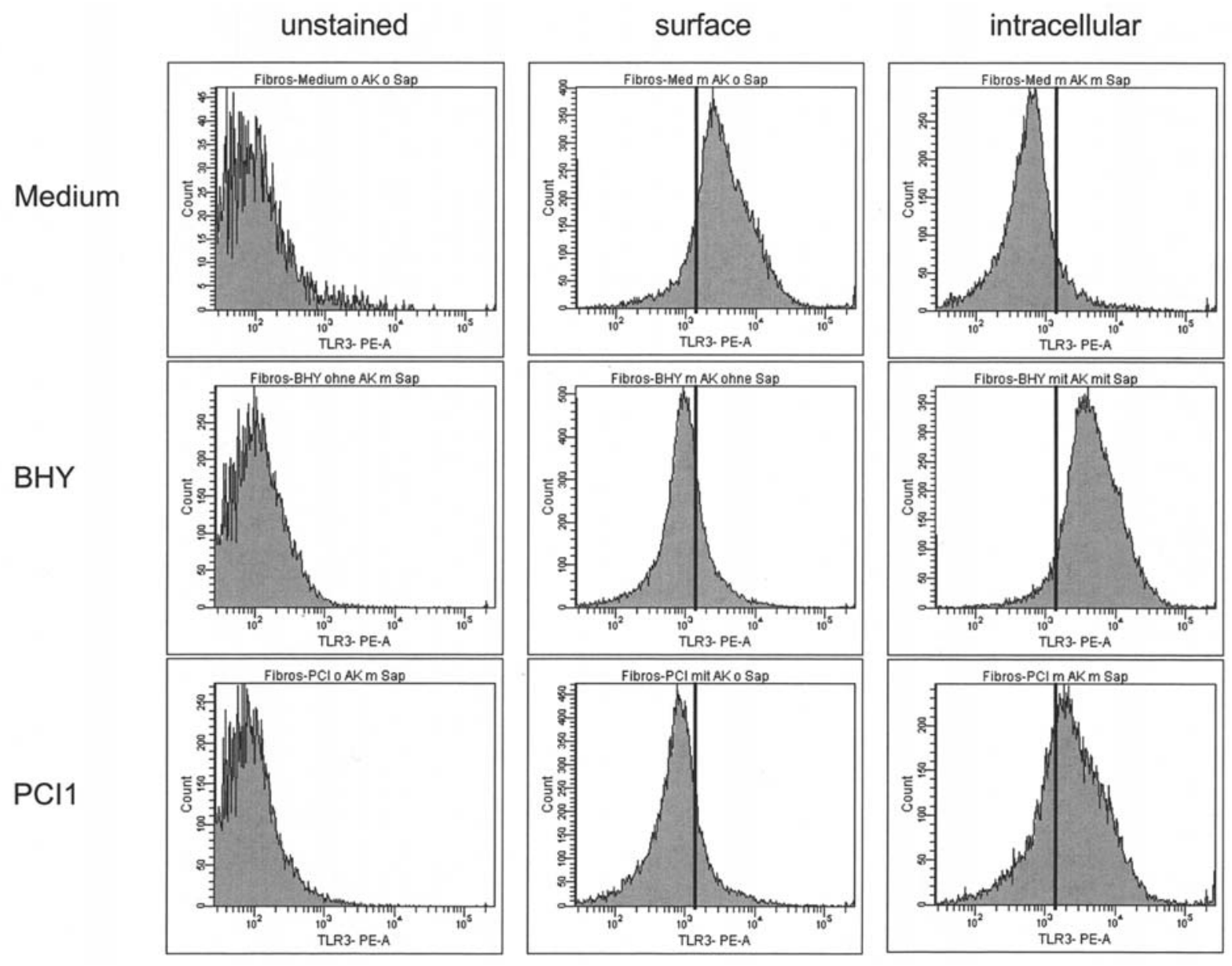

Figure 4. HNSCC-induced internalization of TLR3 in fibroblastic cells. 3T3 fibroblasts expressing heterologous TLR3 showed a decreased expression of TLR3 on the cell surface in response to $24 \mathrm{~h}$ of incubation in HNSCC supernatants of two different HNSCC cell lines (BHY and PCI-1). Correspondingly, increased levels of cytoplasmic TLR3 were observed.

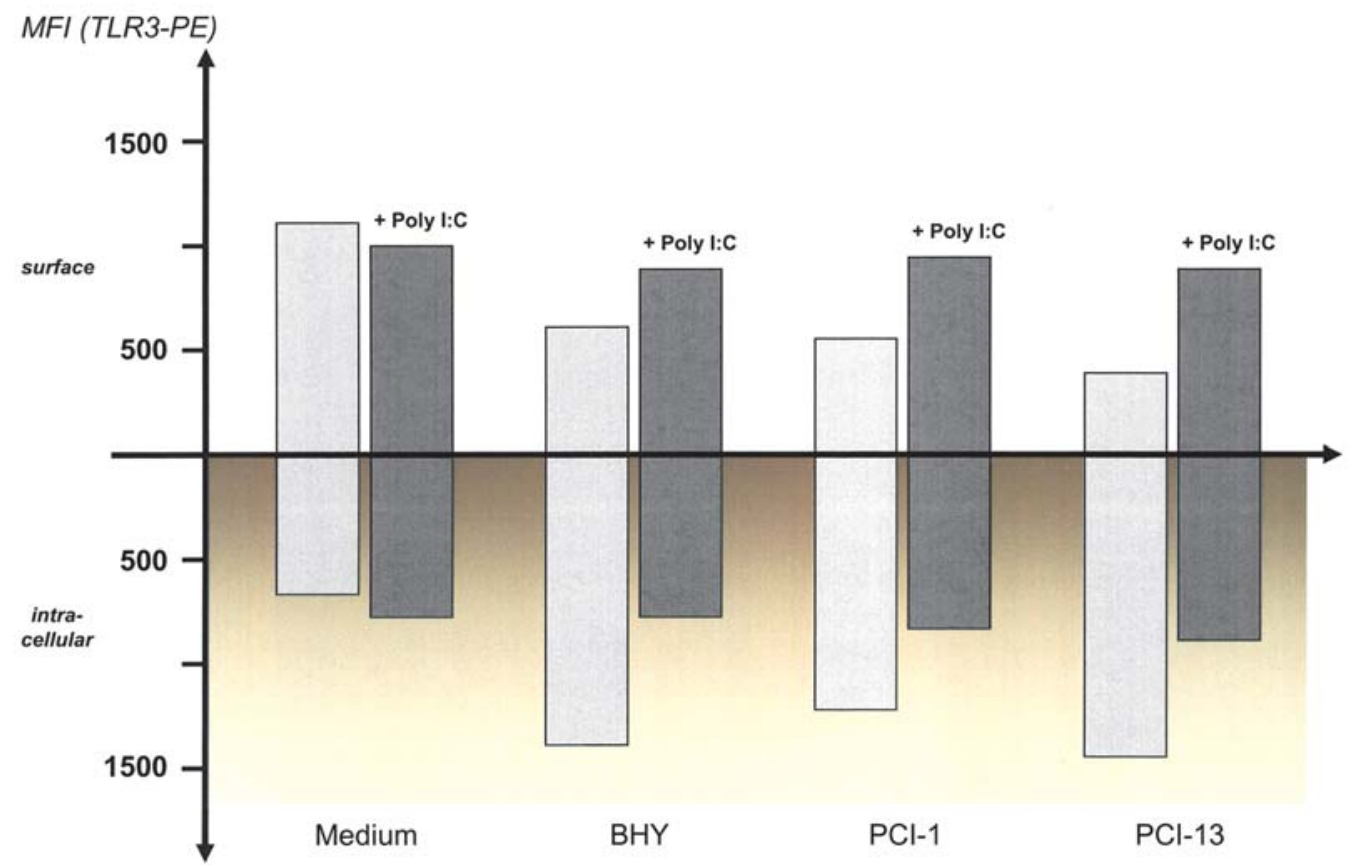

Figure 5. Poly I:C impairs the HNSCC-induced internalization of TLR3. Poly I:C triggers the surface expression of TLR3 in the presence of HNSCC. Poly I:C-stimulated NK cells revealed a significantly increased surface expression of TLR3 in the presence of HNSCC. Supernatants of three different HNSCC cell lines (BHY, PCI-1, and PCI-13) were used. Solid bars illustrate the measured MFI (mean fluorescence intensity). 
A
CD56-PE

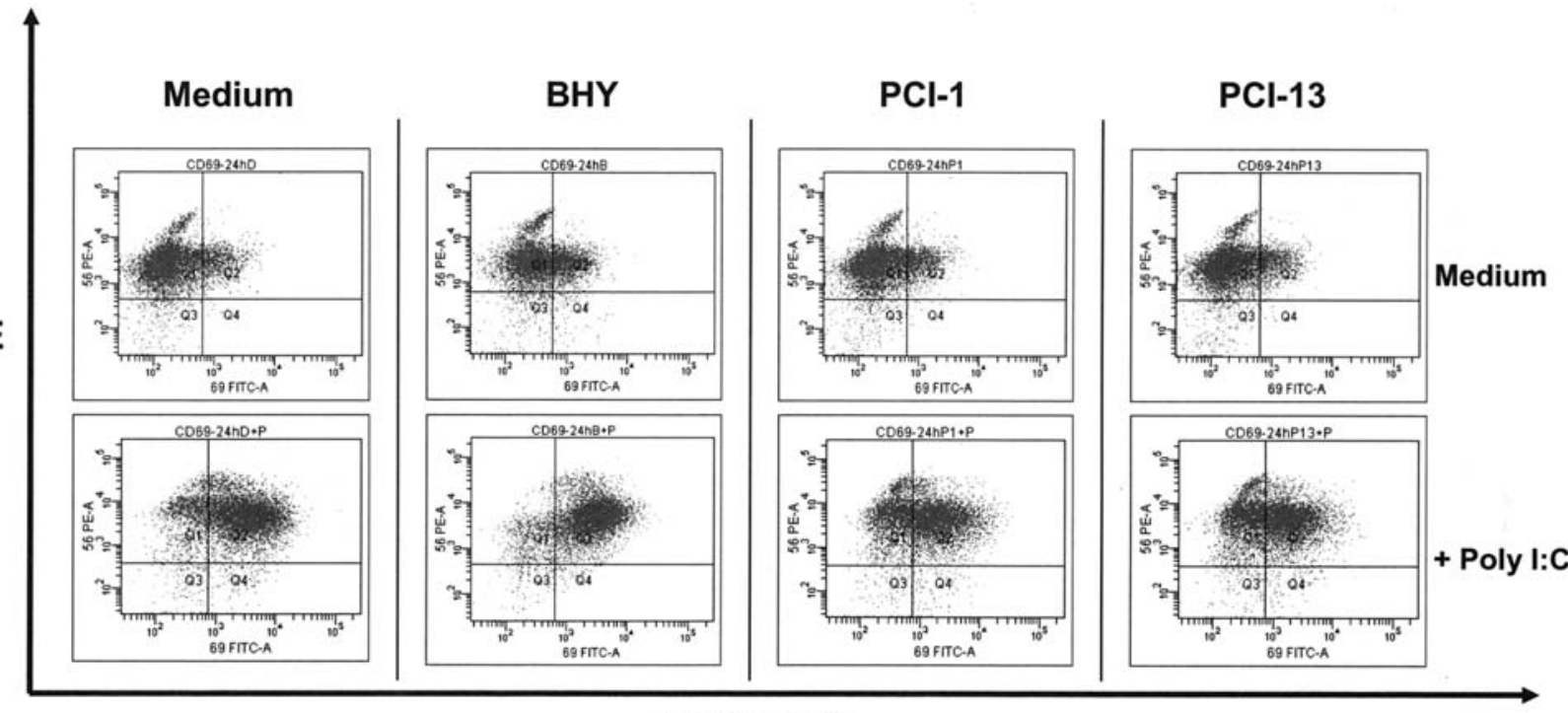

B

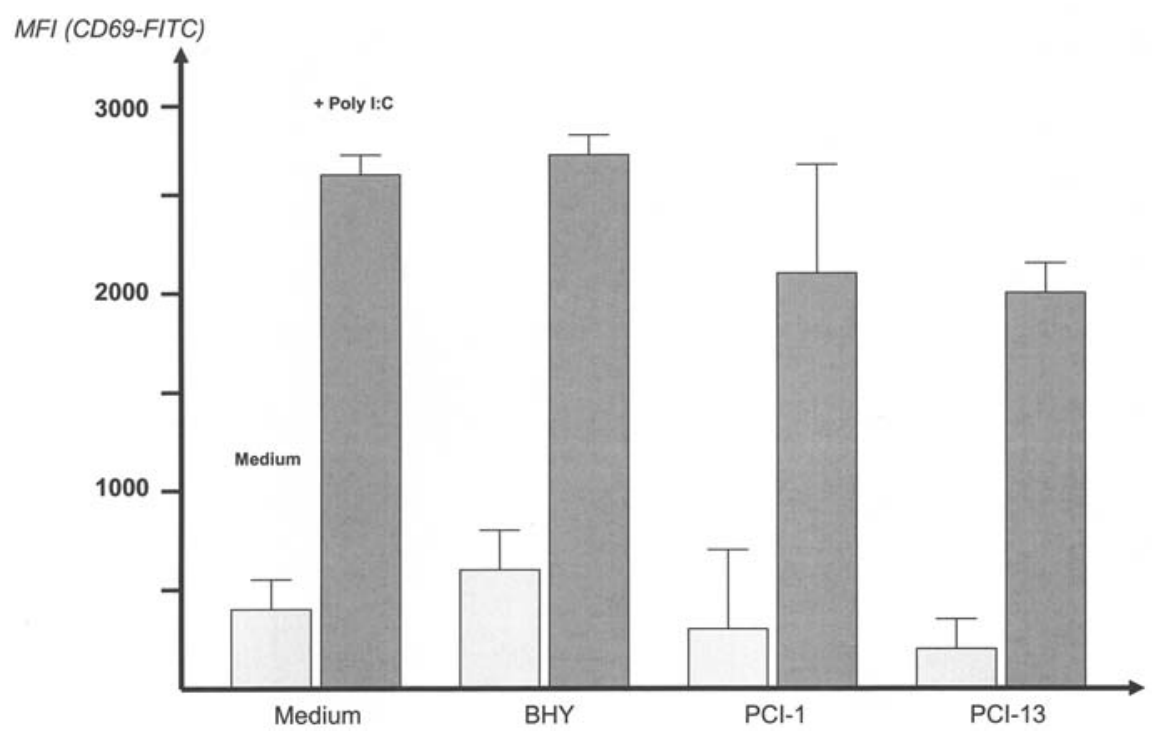

Figure 6. Poly I:C activates human NK cells in the presence of HNSCC. (A) Flow cytometric analysis of activation marker CD69 on human NK cells demonstrated that Poly I:C stimulation resulted in a significant up-regulation of surface CD69, which was not affected by HNSCC supernatants. (B) Relative fluorescence intensity demonstrated the strongly activating capacity of Poly I:C. Supernatants of three different HNSCC cell lines (BHY, PCI-1, and PCI-13) were used. Solid bars illustrate the measured MFI (mean fluorescence intensity).

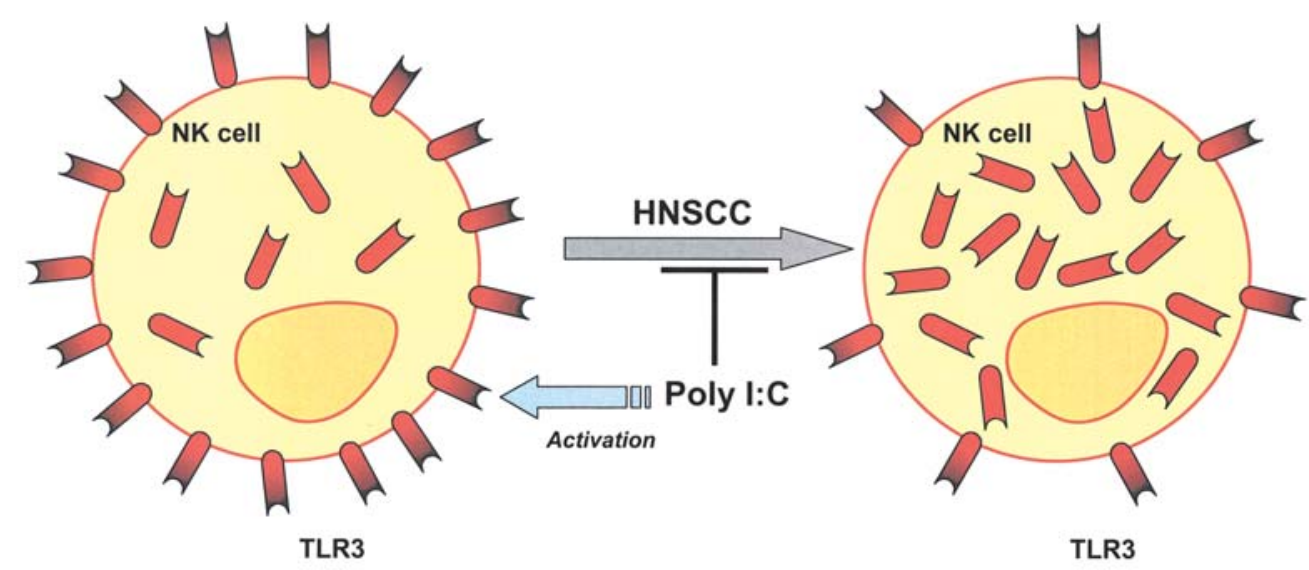

Figure 7. Model of TLR3 internalization. TLR3 was predominantly expressed on the cell surface of NK cells and a smaller protein fraction was also detected within the cellular lumen. HNSCC does not affect the expression level of TLR3, but triggers a rapid internalization of this Toll-like receptor which represents a novel immune-escape mechanism of head and neck cancer. Specific activation with the TLR3 ligand Poly I:C impairs the immune-suppressive influence of HNSCC and results in activated NK cells with predominantly surface-expressed TLR3. 
stimuli such as IL-2, IL-12, IFN- $\alpha$, and Poly I:C (19). Therefore we analyzed the CD69 expression in response to Poly I:C stimulation in the presence and absence of the HNSCC microenvironment.

Our data demonstrated that Poly I:C stimulation resulted in a significant up-regulation of surface CD69 on the analyzed NK cells, which was not significantly affected by HNSCC supernatants (Fig. 5). The influence of head and neck cancer and Poly I:C on TLR3 subcellular localization and the activation of human NK cells is illustrated as a model in Fig. 7.

\section{Discussion}

We investigated the regulation of Toll-like receptor 3 (TLR3) in human NK cells with respect to the influence of head and neck squamous cell carcinoma (HNSCC) and Poly I:C. Besides TLR3, we found that only TLR1, TLR2, and TLR7 were significantly expressed in native NK cells. Flow cytometric investigations revealed that TLR3 was predominantly expressed on the cell surface of natural killer cells. While HNSCC did not affect the protein level of TLR3, our data indicated a rapid internalization of this Toll-like receptor in response to the HNSCC microenvironment resulting in impaired functions. The immune suppressive influence of HNSCC on activation and subcellular distribution of TLR3 was counteracted by specific stimulation with the TLR3 ligand Poly I:C.

Previous investigations regarding the mRNA levels of Toll-like receptors 1-10 in human leukocyte subsets by Northern blot analysis and real-time PCR suggest high expression levels of TLR1, followed by moderate levels of TLR2, TLR3, TLR5, and TLR6 in human NK cells $(20,21)$. We examined the TLR protein levels in NK cells and confirmed the expression of TLR1, TLR2, and TLR3. No significant protein levels of TLR5 and TLR6 were detected, whereas our data indicated the expression of TLR7 in native NK cells. These findings suggest a distinct regulatory mechanism of TLR expression on the transcriptional and translational level, respectively.

Previous publications suggest a cell-specific subcellular localization of TLR3 in different cell types. While human fibroblasts were shown to express TLR3 on the cell surface, investigations with human dendritic cells (DCs) revealed that monocyte-derived immature DCs expressed TLR3 predominantly intracellularly $(22,23)$. Recently it was shown that human lung epithelial cells express TLR3 exclusively in an intracellular compartment and thus was presumed that exogenous dsRNA must be internalized to encounter TLR3 (24).

We found that TLR3 is predominantly expressed on the cell surface of human NK cells, which is rapidly decreased in response to HNSCC. The Toll-like receptor modulating effect of the HNSCC microenvironment was confirmed in fibroblasts expressing heterologous TLR3. These data suggest TLR alterations on tumor-infiltrating leucocytes as an important parameter of the HNSCC immune escape. The responsible mechanisms have to be elucidated in more detail with respect to the development of immunomodulating strategies against HNSCC.

\section{Acknowledgements}

We are grateful to Nicole Bohnert and Brigitte Wollmann for their skillful support in several parts of this study and all members of the Department of Otorhinolaryngology for helpful discussions and a comfortable atmosphere. This study was supported by grants of the following funding bodies: Mildred Scheel-Stiftung (Deutsche Krebshilfe), the MonikaKutzner-Stiftung, the Werner and Klara Kreitz-Stiftung and the Bartling Stiftung.

\section{References}

1. Chin D, Boyle GM, Theile DR, Parsons PG and Coman WB: Molecular introduction to head and neck cancer (HNSCC) carcinogenesis. Br J Plast Surg 57: 595-602, 2004.

2. Douglas WG, Tracy E, Tan D, Yu J, Hicks WL Jr, Rigual NR, Loree TR, Wang Y and Baumann H: Development of head and neck squamous cell carcinoma is associated with altered cytokine responsiveness. Mol Cancer Res 2: 585-593, 2004.

3. Pries R, Thiel A, Brocks C and Wollenberg B: Secretion of tumor-promoting and immune suppressive cytokines by cell lines of head and neck squamous cell carcinoma. In Vivo 20: 45-48, 2006.

4. Trinchieri G: Biology of natural killer cells. Adv Immunol 47: 187-376, 1989.

5. Biron CA, Nguyen KB, Pien GC, Cousens LP and Salazar-Mather TP: Natural killer cells in antiviral defense: function and regulation by innate cytokines. Annu Rev Immunol 17: 189-220, 1999

6. Moretta L, Bottino C, Cantoni C, Mingari MC and Moretta A: Human natural killer cell function and receptors. Curr Opin Pharmacol 1: 387-391, 2001.

7. Biassoni R, Cantoni C, Pende D, Sivori S, Parolini S, Vitale M, Bottino $\mathrm{C}$ and Moretta A: Human natural killer cell receptors and co-receptors. Immunol Rev 181: 203-214, 2001.

8. Moretta A, Bottino C, Vitale M, Pende D, Cantoni C, Mingari MC, Biassoni R and Moretta L: Activating receptors and coreceptors involved in human natural killer cell-mediated cytolysis. Annu Rev Immunol 19: 197-223, 2001.

9. Raulet DH, Vance RE and McMahon CW: Regulation of the natural killer cell receptor repertoire. Annu Rev Immunol 19: 291-330, 2001.

10. Bose A, Ghosh D, Pal S, Mukherjee KK, Biswas J and Baral R: Interferon alpha2b augments suppressed immune functions in tobacco-related head and neck squamous cell carcinoma patients by modulating cytokine signaling. Oral Oncol 42: 161-171, 2006.

11. Wang MB, Lichtenstein A and Mickel RA: Hierarchical immunosuppression of regional lymph nodes in patients with head and neck squamous cell carcinoma. Otolaryngol Head Neck Surg 105: 517-527, 1991

12. Pisegna S, Pirozzi G, Piccoli M, Frati L, Santoni A and Palmieri G: p38 MAPK activation controls the TLR3-mediated upregulation of cytotoxicity and cytokine production in human NK cells. Blood 104: 4157-4164, 2004.

13. Dunne A and O'Neill LA: The interleukin-1 receptor/Toll-like receptor superfamily: signal transduction during inflammation and host defense. Sci STKE, 2003: re3, 2003.

14. Sivori S, Falco M, Della Chiesa M, Carlomagno S, Vitale M, Moretta L and Moretta A: CpG and double-stranded RNA trigger human NK cells by Toll-like receptors: induction of cytokine release and cytotoxicity against tumors and dendritic cells. Proc Natl Acad Sci USA 101: 10116-10121, 2004.

15. Sieling PA and Modlin RL: Toll-like receptors: mammalian 'taste receptors' for a smorgasbord of microbial invaders. Curr Opin Microbiol 5: 70-75, 2002.

16. Kawamata H, Nakashiro K, Uchida D, Harada K, Yoshida H and Sato M: Possible contribution of active MMP2 to lymphnode metastasis and secreted cathepsin $L$ to bone invasion of newly established human oral-squamous-cancer cell lines. Int J Cancer 70: 120-127, 1997.

17. Sozzani S, Luini W, Borsatti A, Polentarutti N, Zhou D, Piemonti L, D'Amico G, Power CA, Wells TN, Gobbi M, Allavena P and Mantovani A: Receptor expression and responsiveness of human dendritic cells to a defined set of CC and CXC chemokines. J Immunol 159: 1993-2000, 1997. 
18. Lanier LL, Testi R, Bindl J and Phillips JH: Identity of Leu-19 (CD56) leukocyte differentiation antigen and neural cell adhesion molecule. J Exp Med 169: 2233-2238, 1989.

19. Gerosa F, Tommasi M, Benati C, Gandini G, Libonati M, Tridente G, Carra G and Trinchieri G: Differential effects of tyrosine kinase inhibition in CD69 antigen expression and lytic activity induced by rIL-2, rIL-12, and rIFN-alpha in human NK cells. Cell Immunol 150: 382-390, 1993.

20. Hornung V, Rothenfusser S, Britsch S, Krug A, Jahrsdorfer B, Giese T, Endres S and Hartmann G: Quantitative expression of toll-like receptor 1-10 mRNA in cellular subsets of human peripheral blood mononuclear cells and sensitivity to $\mathrm{CpG}$ oligodeoxynucleotides. J Immunol 168: 4531-4537, 2002.

21. Muzi M, Bosisio D, Polentarutti N, D'Amico G, Stoppacciaro A Mancinelli R, van't Veer C, Penton-Rol G, Ruco LP, Allavena P and Mantovani A: Differential expression and regulation of tolllike receptors (TLR) in human leukocytes: selective expression of TLR3 in dendritic cells. J Immunol 164: 5998-6004, 2000.
22. Matsumoto M, Funami K, Tanabe M, Oshiumi H, Shingai M, Seto Y, Yamamoto A and Seya T: Subcellular localization of Toll-like receptor 3 in human dendritic cells. J Immunol 171: 3154-3162, 2003

23. Matsumoto M, Kikkawa S, Kohase M, Miyake K and Seya T: Establishment of a monoclonal antibody against human Tolllike receptor 3 that blocks double-stranded RNA-mediated signaling. Biochem Biophys Res Commun 293: 1364-1369, 2002.

24. Guillot L, Le Goffic R, Bloch S, Escriou N, Akira S, Chignard M and Si-Tahar M: Involvement of toll-like receptor 3 in the immune response of lung epithelial cells to double-stranded RNA and influenza A virus. J Biol Chem 280: 5571-5580, 2005. 